\title{
Effects of affective valence on a mixed Spatial Correspondence Task: a reply to Proctor (2013)
}

\author{
Erick Q. Conde ${ }^{1}$, Mikael Cavallet ${ }^{2}$, Nelson Torro-Alves ${ }^{3}$, Elton H. Matsushima ${ }^{4}$, Roberto S. \\ Fraga-Filho $^{4}$, Fernanda Jazenko ${ }^{4}$, Geraldo Busatto ${ }^{2}$, and Luiz G. Gawryszewski ${ }^{4}$ \\ 1- Universidade Federal de Pernambuco, Recife, PE, Brazil \\ 2- Universidade de São Paulo, São Paulo, SP, Brazil \\ 3- Universidade Federal da Paraíba, João Pessoa, PB, Brazil \\ 4- Universidade Federal Fluminense, Rio de Janeiro, RJ, Brazil
}

\begin{abstract}
The present study investigated the influence of emotional valence on the spatial stimulus-key location correspondence effect in three experiments using the Affective Spatial Correspondence task (AffSCt). We initially reanalyzed the results of Conde et al. (2011) according to the model proposed by Proctor (2013). In that study, compatible and incompatible responses were chosen according to the participants' team preference. In one block, the volunteers had to press a key on the same side for the Favorite team and on the opposite side for the Rival team. In another block, a reverse code was used. We found that responses were faster for the Favorite-compatible/Rival-incompatible condition (614 ms) compared with the Favorite-incompatible/ Rival-compatible condition (691 ms). The same experimental arrangement was replicated in another Brazilian city, and similar results were found. Additionally, we employed non-affective "fake" soccer teams as a control condition, and no mappingrule effect was observed. Finally, a final experiment that used the same design but different non-affective stimuli (yellow and blue bars) was performed to provide further evidence that the valence effect in the present experimental paradigm only occurs with affective stimuli. As expected, non-affective stimuli did not produce an overall advantage for any mapping rules, corroborating earlier findings with similar mixed designs. The results confirmed the previous findings and validity of the AffSCt as a methodology to investigate the effects of emotional valence on stimulus-response correspondence. However, we are unable to provide a conclusive explanation to support the several hypotheses proposed previously in our paper and by Proctor (2013). Keywords: spatial correspondence effect, affective valence, soccer preferences, emotion, motor control.
\end{abstract}

Received 16 October 2013; received in revised form 13 May 2014; accepted 27 May 2014. Available online 27 June 2014.

\section{Introduction}

Recent research has shown that the affective features of a stimulus may have a significant influence on both sensory-motor integration and cognitive processing (van Peer, Rotteveel, Spinhoven, Tollenaar, \& Roelofs, 2010). In a previous paper, we proposed a modified version of the spatial compatibility task to study the influence of the affective valence of stimuli on the spatial compatibility

Erick Quintas Conde, Federal University of Pernambuco. Mikael Cavallet and Geraldo Busatto, University of São Paulo. Nelson Torro-Alves, Federal University of Paraíba. Graduate Program in Cognitive Neuroscience and Behavior. Elton Hiroshi Matsushima, Roberto S. Fraga-Filho Fernanda Jazenko, and Luiz G Gawryszewski, Fluminense Federal University. Instituto de Biologia, Neuroscience Program. Correspondence regarding this article should be directed to: Nelson TorroAlves, Universidade Federal da Paraíba, Departamento de Psicologia, Rua Severino Massa Spinelli, 381, Ap. 1703, João Pessoa, 58039-210, Brazil. Phone: 55-83-3216-7337 Fax: 5583-3216-7064. E-mail: nelsontorro@yahoo.com.br effect (Conde et al., 2011). In this new paradigm, which was called the Affective Spatial Correspondence task (AffSCt; Conde et al., 2014), three tasks were combined: (1) the Spatial Compatibility task (which involves an explicit stimulus location code; for review, see Proctor $\& \mathrm{Vu}, 2006),(2)$ the Simon task (based on an implicit spatial location code of neutral stimuli; for review, see Lu \& Proctor, 1995), and (3) the Affective Simon task (which involves an implicit spatial location code for affective stimuli; Zhang \& Proctor, 2008).

In the study by Conde et al. (2011), figures of soccer players of the participants' Favorite and Rival teams were used as positive and negative valence stimuli, respectively. In one block of trials, the participants responded by pressing a key located on the same side for the Favorite team (compatible response) and a key located on the opposite side for the Rival team (incompatible response). In another block, the reverse code was used. We found an ordinary spatial compatibility effect for the Favorite team but a reversed spatial compatibility effect for the Rival team (i.e., the incompatible responses were faster than 
the compatible responses). In that work, we proposed that this modulation would result from approach and withdrawal behaviors toward the Favorite team and away from the Rival team, respectively, which reversed the spatial compatibility effect for the aversive (Rival) team while preserving the compatibility effect for the Favorite team. This statement is supported by the literature, which shows that stimulus valence is linked to withdrawal and approach behavior (Alves, Fukusima, \& AznarCasanova, 2008; Alves, Aznar-Casanova, \& Fukusima, 2009; Markman \& Brendl, 2005; Proctor \& Zhang, 2010) and that people respond faster with approach responses to positive stimuli and withdrawal responses for negative stimuli compared with the opposite mapping (Chen \& Bargh, 1999; De Houwer, Crombez, Baeyens, \& Hermans, 2001; Zhang \& Proctor, 2008)

Freina, Baroni, Borghi, and Nicoletti (2009) proposed that approach and withdrawal reactions are influenced by hand posture. In their study, the participants had to classify the valence of words (positive or negative) by pressing one of two buttons, one located near the body and the other located far from the body. When the participants responded with the open hand, they verified that reaction times were shorter for the far button for positive words and the near button for negative words, in agreement with other studies in the field. However, when the participants had to press the button while holding a tennis ball in their hand, the results were inverted, with participants responding faster with the near button to positive words and the far button to negative words. In the latter case, the participants would be simulating an action of bringing something good to them (approach) and pushing away something bad (avoidance). Despite inversion of the movement, the participants performed actions that were compatible with approach/withdrawal reactions in both cases.

Furthermore, theories of motor control propose that motor planning involves the estimation of costs and rewards associated with the stimulus with which one will interact and monitoring the motor output for future predictions of changes in body states and the immediate environment (de Oliveira et al., 2012). Experimental evidence indicates that sensorimotor cortex activity that precedes the grasping of a stimulus is affected by its valence, with smaller readiness potential amplitudes for grasping pleasant stimuli than for grasping unpleasant stimuli. Smaller potentials were proposed to be related to the recruitment of preset motor repertoires, whereas higher amplitudes that are found for unpleasant stimuli would be attributable to a discrepancy between the required action and its aversiveness (de Oliveira et al., 2012).

Although the approach and withdrawal model would be a plausible explanation for the modulation of Manual Reaction Times (MRTs) by emotional pictures in the AffSCt, other accounts are also possible. In a recent paper, Proctor (2013) considered our approach a valuable tool for studying the effects of affective valence on motor control but proposed a different interpretation of our findings that did not involve a reversal of the spatial compatibility effect. He argued that our experiment was a variant of the mixed task (Shaffer, 1965) in which participants are instructed to make spatially incompatible responses in some trials and spatially compatible responses in others. More specifically, Shaffer (1965) showed that when the correct response to a peripheral target depends on both the target location and a central cue presented at the fixation point (e.g., a horizontal or vertical bar), no difference is found between the compatible condition (target and response key on the same side) and incompatible condition (target and response keys on opposite sides), coded by horizontal and vertical central bars, respectively. Moreover, the response latency increased by approximately $300 \mathrm{~ms}$ when a mapping rule determined the correct response compared with both the compatible block-fixed condition and incompatible block-fixed condition. These results indicate that a super-ordinate system that is related to executive function controls the execution of the correct response in a mixed condition. Thus, based on the results of Shaffer (1965) and those of similar experiments (for review, see Proctor, 2013), Proctor (2013) criticized our interpretation and proposed that our findings were a combined effect that was attributable to the effect of affective valence between blocks of trials. That is, affective valence increases response times when a volunteer performs a non-corresponding response (e.g., stimulus and key on opposite sides) to a positive stimulus (e.g., Favorite team) and a corresponding response (e.g., stimulus and key on the same sides) to a negative stimulus (e.g., Rival team) in a specific block of trials compared with the opposite condition (i.e., corresponding response to the Favorite team and noncorresponding response to the Rival team).

More specifically, when the Favorite team signaled the compatible mapping rule and the Rival team signaled the incompatible mapping rule, MRTs were shorter $(614 \mathrm{~ms})$ than the contrary situation when the Rival team signaled the compatible mapping rule and the Favorite team signaled the incompatible mapping rule (691 ms). In short, our data cannot be explained by an inversion of the spatial compatibility effect for the Rival team because when compatible and incompatible conditions are mixed within a block of trials, the classical spatial compatibility effect vanished (Shaffer, 1965). Nevertheless, it is worth noticing the effect of affective valence between the blocks of trials.

We agree with the new interpretation of Proctor (2013), but an important question remains unanswered. What is the source for the significant differences between the two mapping-rule setups, namely, corresponding and non-corresponding tasks based on team preference?

In the present study we conducted two experiments to test the hypothesis that the affective valence of stimuli interacts with mapping rules. In Experiment 1, we employed the same experimental setting as Conde et al. (2011). We also used non-affective stimuli (i.e., 
"fake" soccer team players) to provide a control for the affective valence effect. In Experiment 2, different nonaffective stimuli (i.e., yellow and blue bars) were tested to confirm the absence of a mapping-rule effect with non-affective stimuli.

To clarify the previous results, we first present a reanalysis of the results of Conde et al. (2011) according to the model proposed by Proctor (2013) and then report the two new experiments.

\section{Reanalysis of the Results of Conde et al. (2011)}

To understand the differences between our interpretations and Proctor's (2013) interpretations, we describe our results according to the two mixed-mapping conditions procedure. The analysis used the same MRT data reported by Conde et al. (2011). Mean MRTs were subjected to three-way repeated-measures analysis of variance (ANOVA) under the following experimental design: 2 Mapping-rules (Favorite-compatible/ Rival-incompatible and Rival-compatible/Favoriteincompatible) $\times 2$ Preferences (Favorite and Rival teams) $\times 2$ Response keys (Left and Right). This analysis revealed a significant main effect of only Mapping-rules $\left(F_{1,13}=6.601, p=.02\right)$, in which the Favorite-compatible/ Rival-incompatible condition (614 ms) was faster than the Favorite-incompatible/Rival-compatible condition (691 ms). This new statistical analysis is correct because it is consistent with the mapping-rule proposal of Proctor (2013) and does not contradict our previous account based on the approach/withdrawal model. In this model, an approach reaction to the positive stimulus (Favorite team) would combine with a withdrawal reaction to the negative stimulus (Rival team), thereby facilitating the responses. In the opposite condition, an inhibition elicited by an instruction-based withdrawal reaction to the positive stimulus (Favorite team) would add up to an instruction-based approach reaction to the negative stimulus (Rival team), delaying the response execution. Moreover, the absence of a Mapping rule $\times$ Preference interaction suggests that the facilitatory effect for compatible responses that were elicited by the positive valence of the Favorite team had the same magnitude as the facilitatory effect elicited in the opposite direction because of the negative valence of Rival team. A similar argument may be considered for the inhibition involved in incompatible responses for the Favorite team and compatible responses for the Rival team.

\section{Experiment 1}

To reinforce the findings reported by Conde et al. (2011), another experiment was conducted with the popular soccer teams and subjects from another city in Brazil. Furthermore, a simple and straightforward approach is to use neutral, non-affective stimuli in the same experimental design to investigate whether the advantage of Favorite-compatible/Rival-incompatible mapping is produced by positive and negative affective valence. By using neutral stimuli, one might expect to find no spatial stimulus-response correspondence (SRC) effect as in similar mixed-mapping tasks (Proctor, 2013; Shaffer, 1965) and also no differences between blocks of trials. The participants in Experiment 1 performed an experimental session with "fake" neutral control teams and another separate experimental session with the Favorite/Rival teams.

\section{Methods}

\section{Participants}

Eight students from the Universidade de São Paulo (Ribeirão Preto campus), aged 18-32 years (seven males and one female), participated in two separate experimental sessions. All of them were right-handed, had normal or corrected-to-normal visual acuity, and were naive about the purposes of the experiment.

\section{Apparatus and stimuli}

The experimental sessions were performed in a dimly lit room where the participant sat in front of a monitor with his/her head positioned in a head support at a viewing distance of $60 \mathrm{~cm}$. Responses were made with the index fingers positioned on the left and right trigger buttons, respectively, of a joystick (Microsoft Sidewinder Game Pad). The stimuli were presented on a 17 -inch color monitor with $1024 \times 768$ pixel resolution and a $100 \mathrm{~Hz}$ refresh rate. Stimulus presentation and response recording were performed using E-Prime 2.0 software (Psychology Software Tools, Pittsburgh, PA, USA). In one experimental session, the stimuli were two colored (blue and white) figures $\left(6.5^{\circ}\right.$ height $\times 2.3^{\circ}$ width) of "fake" soccer teams (Striped and Squared) that represented non-affective stimuli. In the other experimental session, four stimuli that represented the four most popular soccer teams in São Paulo, Brazil (Santos, Palmeiras, São Paulo, and Corinthians) were used. The stimuli are shown in Figure 1. The stimuli were randomly presented at a visual angle of $6.7^{\circ}$ to the left or right of a centered fixation stimulus.

\section{Procedure}

Initially, each participant ranked their order of preference for the four soccer teams: Santos, Palmeiras, São Paulo, and Corinthians. The first and fourth teams, in order of preference, defined the Favorite and Rival soccer teams for each participant. This assessment determined which stimuli that were presented in the task were regarded as the Favorite team (positive valence) and Rival team (negative valence) by the participants (Conde et al., 2011).

Subsequently, the participants began an experimental session that was composed of two counterbalanced blocks of trials with the "fake" soccer teams (Striped and Squared). In one block, the participants were instructed to respond by pressing the key on the same side for the Striped stimulus (compatible condition) and pressing the key on the opposite 


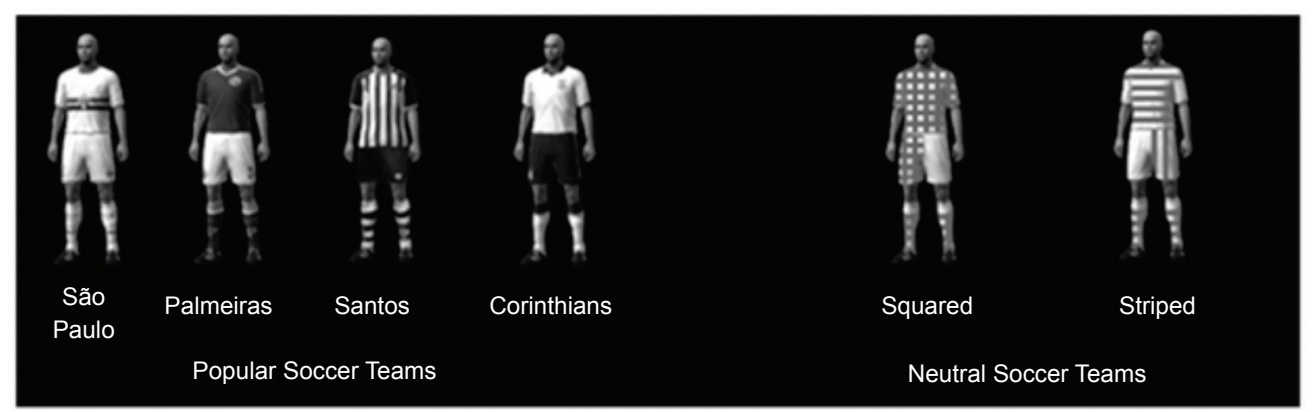

\section{EXPERIMENT 1}

Block A

For the Favorite team: press the key located at the same side. For the Rival team: press the key located on the opposite side.

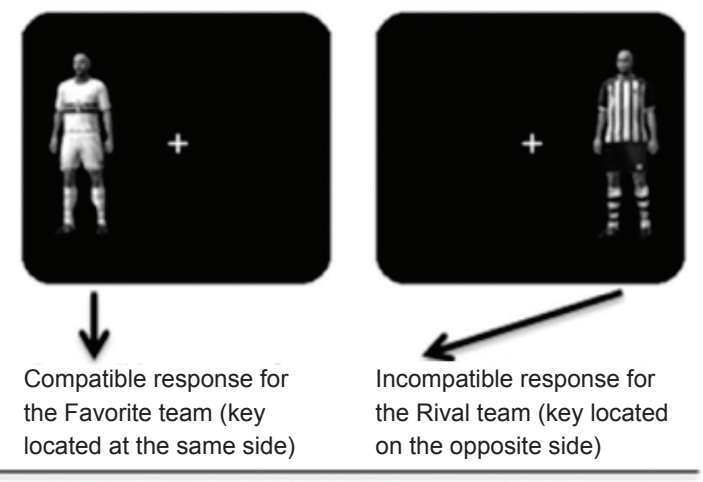

\section{Block B}

For the Rival team: press the key located at the same side. For the Favorite team: press the key located on the opposite side.

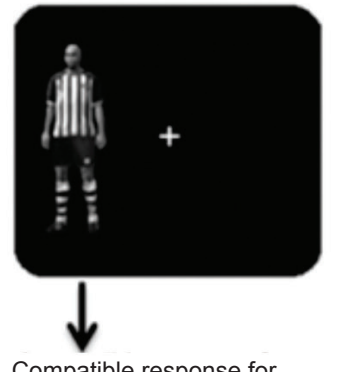

Compatible response for the Rival team (key located at the same side)

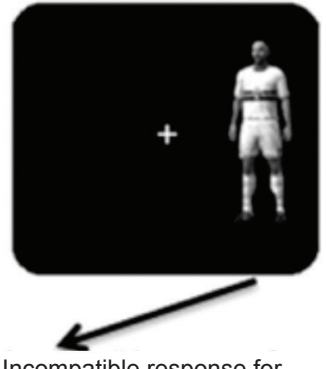

Incompatible response for the Favorite team (key located on the opposite side)

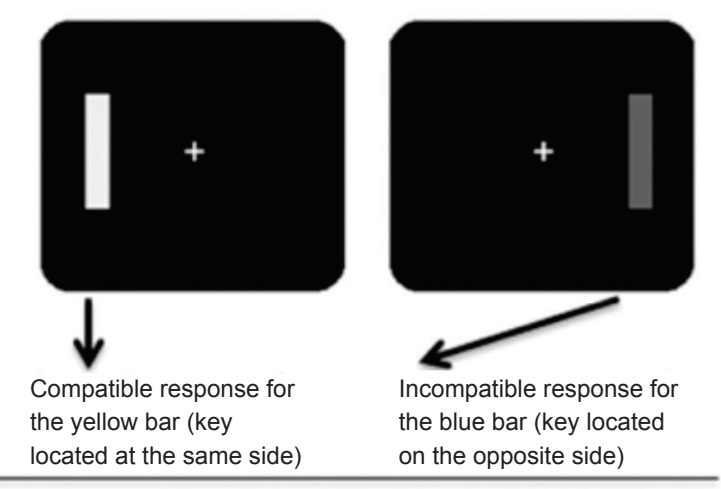

Block B

For the blue bar: press the key located at the same side.

For the yellow bar: press the key located on the opposite side.

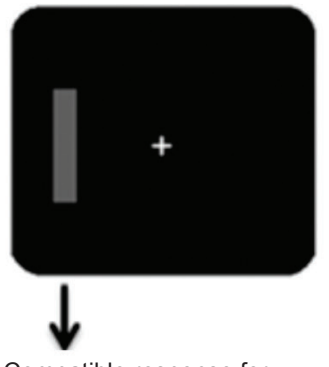

Compatible response for the blue bar (key located at the same side)

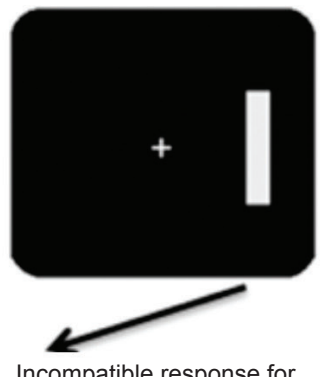

Incompatible response for the yellow bar (key located on the opposite side)

Figure 1. Stimuli used in Experiment 1 (top) and the compatible and incompatible conditions in Experiments 1 and 2 (left and right panels, respectively). In Block A, the participants responded to the Favorite team (Experiment 1) and Yellow bar (Experiment 2) by pressing the key located on the same side and responded to the Rival team (Experiment 1) and Blue bar (Experiment 2) by pressing the key located on the opposite side. In both experiments, the mapping of responses was reversed in Block B.

side for the Squared stimulus (incompatible condition). In the other block, reverse mapping instructions were given (i.e., the compatible condition for the Squared stimulus and incompatible condition for the Striped stimulus).

The participants then underwent a second experimental session that was the same as the first session, with the exception of presentation of the Favorite and Rival soccer teams. Therefore, in one block, the participants were instructed to respond by pressing the key on the same side for the Favorite team (compatible condition) and pressing the key on the opposite side for the Rival team (incompatible condition). In the other block, reverse mapping instructions were given (i.e., the compatible condition for the Rival team and incompatible condition for the Favorite team).

Each block began with 24 training trials followed by 80 test trials. At the end of each training trial, feedback was given. Each trial began with the presentation of the fixation stimulus ("+" sign) for $500 \mathrm{~ms}$, followed by presentation of the soccer team for $700 \mathrm{~ms}$. After the participant's response, the next trial began after an interval of $1300 \mathrm{~ms}$. 


\section{Results}

Importantly, no affective valence was linked to each of the "fake" soccer teams. As a corollary, no Favorite and Rival teams were included in this step of the experiment. The "fake" team blocks always preceded the real team blocks, implying "training" bias in favor of the experiment with the real soccer teams, thus preventing direct comparisons between the "fake" and Favorite/Rival team experimental sessions. Based on these considerations, we decided to perform two ANOVAS, one for the Favorite/Rival soccer teams (a replication of our previous experiment) and another for the "fake" teams.

\section{Favorite/Rival team experimental session}

The mean MRTs for the Favorite/Rival teams were subjected to a three-way repeated-measures ANOVA with the following experimental design: 2 Mapping-rules (Favorite-compatible/Rival-incompatible and Rivalcompatible/Favorite-incompatible) $\times 2$ Preferences (Favorite and Rival teams) $\times 2$ Response keys (Left and Right). The analysis revealed a significant main effect of only Mapping-rules $\left(F_{1,7}=8.48, p=.02\right)$, in which the Favorite-compatible/Rival-incompatible condition (530 ms) was faster than the Favorite-incompatible/ Rival-compatible condition (572 ms; Figure 2).

\section{"Fake" team experimental session}

The mean MRTs for the "fake" teams were subjected to a three-way repeated-measures ANOVA with the following experimental design: 2 Mappingrules (Striped-compatible/Squared-incompatible and Squared-compatible/Striped-incompatible) $\times 2$ Fake soccer teams (Striped and Squared teams) $\times 2$ Response keys (Left and Right). The analysis revealed no significant main effects of Mapping rules $\left(F_{1,7}=.88, p=.37\right)$, Fake soccer teams $\left(F_{1,7}=1.21, p=.30\right)$, or Response keys $\left(F_{1,7}\right.$ $=1.18, p=.31)$ and no two- or three-way interactions $(p$ $>.05$; Figure 2, Table 1).

\section{Discussion}

In the experimental session that used Favorite/Rival soccer teams, we replicated our previous findings in which there was an advantage for the Favorite-compatible/ Rival-incompatible condition (530 ms) compared with the Favorite-incompatible/Rival-compatible condition ( $572 \mathrm{~ms}$ ). These results reinforce the findings reported by Conde et al. (2011) and show that the difference between the two blocks of trials with opposite mapping rules can be reproduced with different popular soccer teams from another Brazilian region.

In contrast, when no affective valence was linked to the soccer stimuli (i.e., the "fake" teams), the mapping-rule effect was absent. These results are distinct from both the results reported by Conde et al. (2011) and the results obtained with the Favorite/Rival soccer teams in the present experiment. The absence of an advantage for one of the mapping rules with the "fake" teams contrasts with the faster reactions times obtained for Favoritecompatible/Rival-incompatible mapping compared with Favorite-incompatible/Rival-compatible mapping. This distinction is important because both experiments used the same experimental setup and differed only in the affective valence of the stimuli, which was present for the Favorite and Rival teams. Moreover, as expected, no spatial correspondence effect was observed with the "fake" teams, thus corroborating earlier findings with similar mixed designs (Proctor, 2013; Shaffer, 1965).

\section{Experiment 2}

Although the results of Experiment 1 confirmed previous findings and tested a control situation with "fake" teams, the neutral teams had the same colors and were very similar to each other. Therefore, the participants may not have associated each stimulus with specific spatial mapping, such as what occurred with the Favorite/Rival teams. Although the "fake" stimuli appeared to provide a good control condition, testing the task with different neutral stimuli should enable us to generalize our previous findings. Therefore, we decided to use distinctly colored stimuli as neutral stimuli to complement our experiment using "fake" soccer teams.

In this task, the participants had to respond to colored stimuli that were presented in two blocks of trials: Yellow-compatible/Blue-incompatible and Bluecompatible/Yellow-incompatible. We expected to find no differences between the blocks of trials and no spatial SRC effect, such as what was observed with the "fake" stimuli.

\section{Methods}

\section{Participants}

Sixteen students from the Universidade Federal Fluminense (Rio de Janeiro, Brazil), aged 19-30 years (nine males and seven females), participated in the experiment after providing written informed consent. All of them were right-handed (Oldfield, 1971), had normal or corrected-to-normal visual acuity, and were naive about the purposes of the experiment. The study was approved by the Institutional Ethics Commission of the Universidade Federal Fluminense (process no. $185 / 2005)$.

\section{Apparatus and stimuli}

The experiment was performed in a dimly lit and soundproof chamber where the participants sat in front of a monitor at a viewing distance of approximately $57 \mathrm{~cm}$. All of the responses were made with the index fingers of the left and right hands using a left key (the letter " $A$ ") and a right key (the number " 6 " on the numerical keypad) on a 

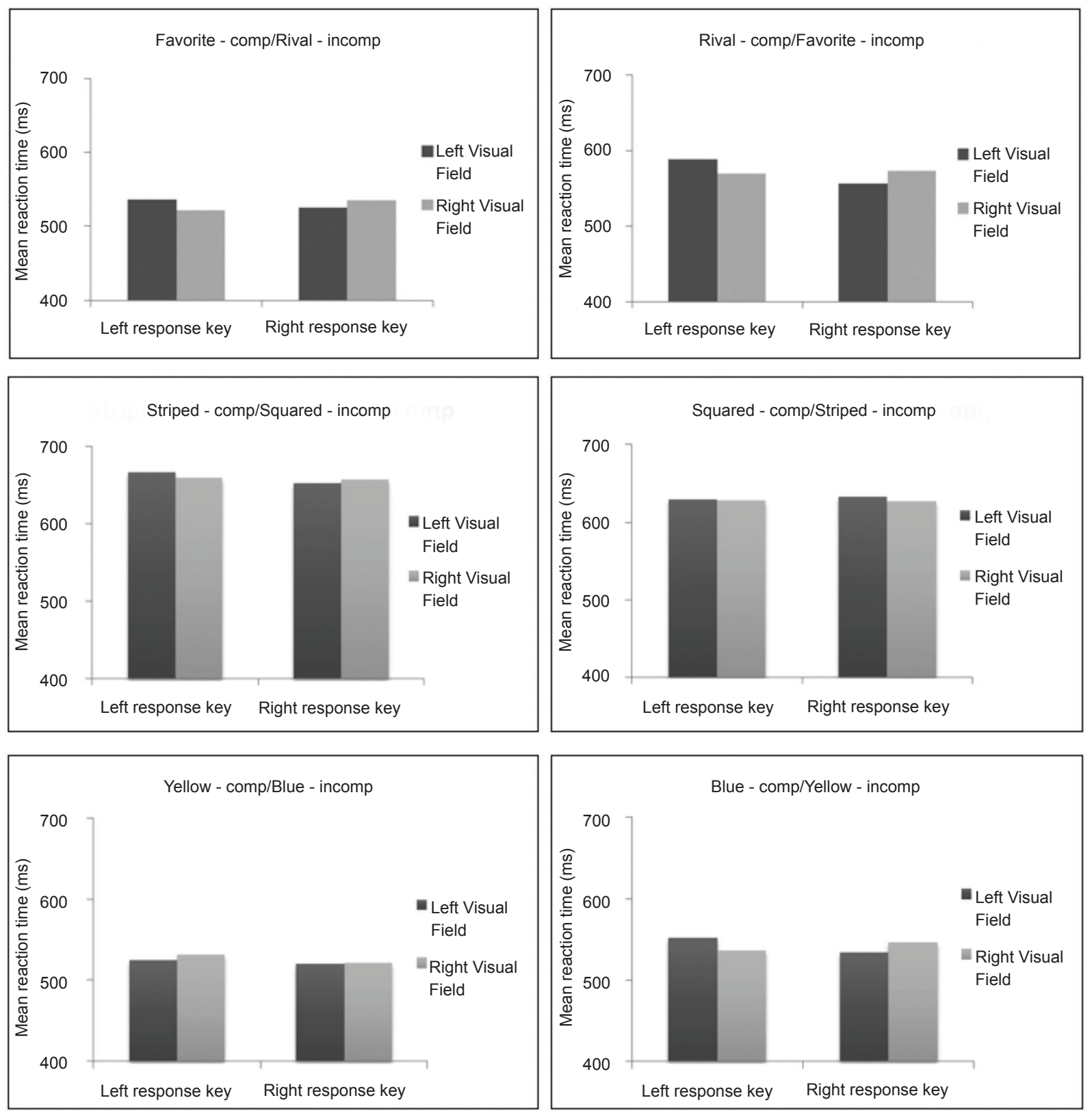

Figure 2. Mean manual response times in the two experiments for each mapping condition as a function of the response key and type of stimulus (team preference, non-affective teams, and color). Comp, compatible; Incomp, incompatible.

computer keyboard. The stimuli were vertical yellow and blue bars $\left(1.5^{\circ}\right.$ width $\times 6.5^{\circ}$ height $)$ and randomly presented $6.0^{\circ}$ to the left or right of a fixation point. E-Prime 2.0 software was used to present the stimuli and record MRTs.

\section{Procedure}

The experimental session consisted of two counterbalanced blocks with 120 trials each. In each experimental block, the stimuli were yellow and blue bars. Before each experimental block, the participants responded in a training block with 40 trials. The participants were instructed to respond by pressing the key located on the same side as the stimulus for one color (corresponding condition) and pressing the key located on the opposite side of the stimulus for the other color (non-corresponding condition). For half of the sample, in the first block, the corresponding condition was used for the yellow bar, and the noncorresponding condition was used for the blue bar. For the other half, in the first block, the corresponding condition was used for the blue bar, and the noncorresponding condition was used for the yellow bar (Figure 1). In the second block, the corresponding conditions for the colors were reversed.

\section{Results}

The data analyses were conducted using PASW Statistics 18.0. Mean MRTs were subjected to a threeway repeated-measures ANOVA with the following experimental design: 2 Mapping rules (Yellow- 
compatible/Blue-incompatible and Blue-compatible/ Yellow-incompatible) $\times 2$ Colors (Yellow and Blue) $\times$ 2 Response keys (Left and Right) as the main withinsubjects factors. This analysis revealed no significant main effects of Mapping rules $\left(F_{1,15}=2.36, p=.14\right)$, Color $\left(F_{1,15}=3.65, p=.08\right)$, or Response keys $\left(F_{1,15}=\right.$ $1.28, p=.28)$ and no two- or three-way interactions $(p>$ .05 ; Figure 2, Table 1 .

\section{Discussion}

The results of Experiment 2 showed no overall advantage of either of the mapping rules (i.e., no difference in performance between the Yellowcompatible/Blue-incompatible and Blue-compatible/ Yellow-incompatible mapping rules as with the "fake" stimuli tested in Experiment 1). The experiment that used yellow and blue bars provided a good neutral control condition because it eliminated any doubt about the effects of affective valence on the AffSCt with soccer stimuli.

\section{General discussion}

In the present study, we first reanalyzed the results of Conde et al. (2011) by considering Proctor's criticism of our interpretation. We then replicated our previous findings in another Brazilian city that were initially observed with Rio de Janeiro teams. Finally, we conducted two experiments to test the experimental procedure with neutral non-affective stimuli ("fake" soccer teams and colored bars).

Table 1. Detailed results obtained for each mapping and experimental condition.

\begin{tabular}{|c|c|c|c|c|}
\hline \multirow{2}{*}{ Mapping } & \multirow{2}{*}{$\begin{array}{c}\text { Visual } \\
\text { Field }\end{array}$} & \multicolumn{2}{|c|}{ Response Key } & \multirow{2}{*}{$\begin{array}{l}\text { Mapping } \\
\text { Average (SE) }\end{array}$} \\
\hline & & Left & Right & \\
\hline \multirow{2}{*}{$\begin{array}{l}\text { Favorite } \rightarrow \text { Comp/Ri- } \\
\text { val } \rightarrow \text { Incomp }\end{array}$} & Left & 537 & 526 & \multirow{2}{*}{$\begin{array}{c}530.25 \\
(15.350)\end{array}$} \\
\hline & Right & 522 & 536 & \\
\hline \multirow{2}{*}{$\begin{array}{l}\text { Rival } \rightarrow \text { Comp } / \\
\text { Favorite } \rightarrow \text { Incomp }\end{array}$} & Left & 589 & 556 & \multirow{2}{*}{$\begin{array}{c}572.25 \\
(18.745)\end{array}$} \\
\hline & Right & 570 & 574 & \\
\hline \multirow{2}{*}{$\begin{array}{l}\text { Striped } \rightarrow \text { Comp } / \\
\text { Squared } \rightarrow \text { Incomp }\end{array}$} & Left & 667 & 652 & \multirow{2}{*}{$\begin{array}{c}658.75 \\
(20.054)\end{array}$} \\
\hline & Right & 659 & 657 & \\
\hline \multirow{2}{*}{$\begin{array}{l}\text { Squared } \rightarrow \text { Comp } / \\
\text { Striped } \rightarrow \text { Incomp }\end{array}$} & Left & 630 & 633 & \multirow{2}{*}{$\begin{array}{c}629.75 \\
(13.053)\end{array}$} \\
\hline & Right & 629 & 627 & \\
\hline \multirow{2}{*}{$\begin{array}{l}\text { Yellow } \rightarrow \text { Comp/ } \\
\text { Blue } \rightarrow \text { Incomp }\end{array}$} & Left & 524 & 520 & \multirow{2}{*}{$\begin{array}{l}524.38 \\
(11.02)\end{array}$} \\
\hline & Right & 531 & 522 & \\
\hline \multirow{2}{*}{$\begin{array}{l}\text { Blue } \rightarrow \text { Comp } / \\
\text { Yellow } \rightarrow \text { Incomp }\end{array}$} & Left & 552 & 534 & \multirow{2}{*}{$\begin{array}{l}542.21 \\
(11.90)\end{array}$} \\
\hline & Right & 537 & 546 & \\
\hline
\end{tabular}

${ }^{*} p<.02$, significant values for mapping-rule factor. SE, standard error of the mean; Comp, compatible; Incomp, incompatible
In contrast to the results of Experiment 1, we found that the use of "fake" soccer teams and colored bars did not elicit differences between the mapping rules or any SRC effects, corroborating earlier findings with similar mixed designs (Heister \& Schroeder-Heister, 1994; Proctor, 2013; Shaffer, 1965). These results are similar to those reported by Conde et al. (2011) in which no SRC effect was observed, but we must emphasize that the absence of a formal SRC effect in the study by Conde et al. (2011) does not necessarily indicate the absence of a spatial stimulus-key correspondence effect. Indeed, one may consider that a spatial stimuluskey correspondence effect occurred, but it was reversed because of the affective valence of the stimuli, resulting in faster response times for the Favorite team in the corresponding condition and Rival team in the noncorresponding condition. Thus, the difference between the two blocks likely reflected a combined effect of facilitatory and inhibitory influences that cannot be disentangled from the stimuli with emotional valence elicited by preference for the Favorite and Rival soccer teams, respectively.

\section{Methodological implications of our new experiments}

Considering the findings of the present study and those of Conde et al. (2011), we demonstrated that the affective valence of a visual stimulus may have a strong influence on the SRC effect. No effect was observed for non-affective stimuli, but a strong effect was observed for Favorite and Rival teams as tested in the two Brazilian cities.

Altogether, these results indicate that our methodology, which we suggest can be renamed the AffSCt, is sufficiently sensitive for exploring affective influences on spatial SRC. We found mapping-rule effects for Favorite-Rival Team preference, both in Rio de Janeiro and São Paulo, and we believe that the AffSCt may be useful for studying affective antagonism that is inherent to cultural, political, and social human activities.

\section{Concluding remarks}

In his analysis of our results, Proctor (2013) proposed three possible explanations for our soccer team preference effects. First, he considered that compatible and incompatible mappings have positive and negative affective valence, which would overlap along the stimulus and mapping-rule affect dimensions. Second, he suggested that favorite and rival teams should be coded as "compatible" and "incompatible," respectively, so "it is easier to select the appropriate mapping rule when the compatible team is mapped to the compatible rule and the incompatible team to the compatible rule than with the opposite mapping" (Proctor, 2013). Third, teams are not coded as compatible and incompatible, but the members of each dimension are coded asymmetrically as in linguistic marking, with one member dominant or unmarked (Favorite team) and 
the other member marked in relation to it (Rival team), resulting in codes with different polarities (for further details, see Proctor, 2013). Linguistic marking is an emergent field of research in the cognitive neuroscience of spatial metaphors (Schnall \& Clore, 2004; Pecher, van Dantzig, Boot, Zanolle, \& Huber, 2010; Hutchinson \& Lowerse, 2010; van Dantzig \& Pecher, 2011).

Although linguistic marking may have influenced our results, previous studies of affective Simon effects, which showed that MRTs are influenced by the affective valence of the target (De Houwer et al., 2001; Markman \& Brendl, 2005; Zhang \& Proctor, 2008), support our hypothesis that the positive and negative affective valence of the stimuli elicits approach and withdrawal reactions, respectively. Moreover, Freina et al. (2009) reported findings similar to ours using the explicit processing of positive and negative words and responses that were made by pressing near and far keys under conditions that simulated approach and withdrawal reactions. Thus, we propose that approach/ withdrawal reactions should be included in the three explanations proposed by Proctor (2013) to interpret the findings of Conde et al. (2011) and design future experiments. However, the existence or not of approach and withdrawal responses to these emotional stimuli is a relevant issue (and sustained by the literature) that must be further investigated to better understand the mechanisms that affect responses within a mapping-rule approach for the AffSCt.

The experimental AffSCt protocol may be considered an important tool to investigate the influence of emotional pictures in mapping rules, compatibility effects, and other spatially oriented responses. The absence of any significant effect when non-emotional stimuli were presented is the strongest evidence that emotion is a key factor that generates attentional and response bias, independent of whether it is elicited by approach-withdrawal reactions, compatibility effects, or the polarity of codes generated by stimuli and responses.

\section{Acknowledgements}

The authors wish to thank Prof. Cesar A. Galera for providing the equipment and laboratory to run the experiment performed in Ribeirão Preto. We also thank Prof. Proctor for his valuable comments and reappraisal of Shaffer's (1965) paper, which is a seminal article that is usually not cited in papers on spatial compatibility tasks.

CNPq-- Proc. 450588/2009-2, Proc. 309544/2009-2, Proc. 483611/2009-3, Proc. 455353/2012-3, Proc. 312189/2012-5, Proc. 478834/2013-6 CAPES. FAPERJ -- Edital FAPERJ n. ${ }^{\circ}$ 06/2013 (Pró-Idoso) -- Proc. 110.079/2013, E-26/101.958/2010, E-26/101.061/2011, E-26/100.661/2013, E-26/102.160/2013. PIBIC/UFF/ CNPq, PIBITI/UFF/CNPq, FOPESQ/PROPPi/UFF. CNPq - Proc. 479793/2013-1. Fapesp:2011/09946-9 e 2011/19179-5.

\section{References}

Alves, N. T., Aznar-Casanova, J. A., \& Fukusima, S. S. (2009). Patterns of brain asymmetry in the perception of positive and negative facial expressions. Laterality: Asymmetries of Body, Brain and Cognition, 14(3), 256-272.

Alves, N. T., Fukusima, S. S., \& Aznar-Casanova, J. A. (2008). Models of brain asymmetry in the processing of emotions. Psychology \& Neuroscience, 1(1), 63-66.

Chen, M., \& Bargh, J. A. (1999). Consequences of automatic evaluation: immediate behavioral predispositions to approach or avoid the stimulus. Personality and Social Psychology Bulletin, 25(2), 215-224.

Conde, E. F. Q., Jazenko, F., Fraga Filho, R. S., da Costa, D. H., TorroAlves, N., Cavallet, M., \& Gawryszewski, L. G. (2011). Stimulus affective valence reverses spatial compatibility effect. Psychology \& Neuroscience, 4(1), 81-87.

Conde, E. C. Q., Matsushima, E. H., Torro-Alves, N., Cavallet, M., Jazenko, F., Fraga-Filho, R. S., \& Gawryszewski, L. G. (2014). Affective Spatial Compatibility task (AffSCt): theory and applications. Temas em Psicologia, in press.

De Houwer, J., Crombez, G., Baeyens, F., \& Hermans, D. (2001). On the generality of the affective Simon effect. Cognition \& Emotion, 15(2), 189-206.

de Oliveira, L. A. S., Imbiriba, L. A., Russo, M. M., Nogueira-Campos, A. A., Rodrigues, E. D. C., Pereira, M. G., \& Volchan, E. (2012). Preparing to grasp emotionally laden stimuli. PLoS One, 7(9), e45235.

Freina, L., Baroni, G., Borghi, A. M., \& Nicoletti, R. (2009). Emotive concept nouns and motor responses: attraction or repulsion? Memory \& Cognition, 37(4), 493-499.

Heister, G., \& Schroeder-Heister, P. (1994). Spatial S-R compatibility: positional instruction vs. compatibility instructions. Acta Psychologica, 85, 15-24.

Hutchinson, S., \& Louwerse, M. M. (2010). The upbeat of language: linguistic context and embodiment predict processing valence words. Proceedings of the 34th Annual Meeting of the Cognitive Science Society (pp. 1709-1714). Austin, TX: Cognitive Science Society.

Lu, C. H., \& Proctor, R. W. (1995). The influence of irrelevant local information on performance: a review of the Simon effect and spatial attention. Psychonomic Bulletin and Review, 2(2), 174-207.

Markman, A. B., \& Brendl, C. M. (2005). Constraining theories of embodied cognition. Psychological Science, 16(1), 6-10.

Pecher, D., van Dantzig, S., Boot, I., Zanolie, K., \& Huber, D. E. (2010). Congruency between word position and meaning is caused by task-Induced spatial attention. Frontiers in Psychology, 1, 30.

Proctor, R. W. (2013). Stimulus affect valence may influence mappingrule selection but does not reverse the spatial compatibility effect: reinterpretation of Conde et al. (2011). Psychology \& Neuroscience, 6(1), 3-6.

Proctor, R. W., \& Vu, K. P. L. (2006). Stimulus-response compatibility principles: data, theory, and application. Boca Raton, FL: CRC Press.

Proctor, R. W., \& Zhang, Y. (2010). "Mother nature doesn't have a bullet with your name on it": coding with reference to one's name or object location? Journal of Experimental Social Psychology, 46(2), 336-343.

Oldfield, R. C. (1971). The assessment and analysis of handedness: the Edinburgh inventory. Neuropsychologia, 9, 97-113.

Schnall, S., \& Clore, G. L. (2005). Emergent meaning in affective space: conceptual and spatial congruence produces positive evaluations. Proceedings of the 36th Annual Conference of the Cognitive Science Society (p. 1209-1214). Mahwah: Lawrence Erlbaum.

Shaffer, L. H. (1965). Choice reaction with variable S-R mapping. Journal of Experimental Psychology, 70, 284-288.

van Dantzig, S., \& Pecher, D. (2011). Spatial attention is driven by mental simulations. Frontiers in Psychology, 2, 40.

van Peer, J. M., Rotteveel, M., Spinhoven, P., Tollenaar, M. S., \& Roelofs, K. (2010). Affect-congruent approach and withdrawal movements of happy and angry faces facilitate affective categorisation. Cognition \& Emotion, 24(5), 863-875.

Zhang, Y., \& Proctor, R. W. (2008). Influence of intermixed emotionrelevant trials on the affective Simon effect. Experimental Psychology, 55(6), 409-416. 